\title{
Defining the optimal analgesic strategy for erector spinae plane (ESP) blocks in unanticipated open cholecystectomy
}

\author{
Julien Raft ${ }^{1}$, Ki Jinn Chin ${ }^{2}$, Quentin Gobert ${ }^{1}$, Philippe Richebé ${ }^{1}$, and \\ Véronique Brulotte ${ }^{1}$ \\ ${ }^{I}$ Department of Anesthesiology and Pain Medicine, University of Montréal, Maisonneuve-Rosemont Hospital, \\ Montréal, QC, ${ }^{2}$ Department of Anesthesia and Pain Medicine, Toronto Western Hospital, University of \\ Toronto, Toronto, ON, Canada
}

Laparoscopic cholecystectomy (LC) is the standard operative procedure for cholelithiasis, but the reported conversion rate to open cholecystectomy (OC) is $4.8 \%$ or higher [1]. Postoperative pain after OC is significantly greater than after LC and regional anesthesia techniques are useful for improving analgesia and recovery [2]. The erector spinae plane (ESP) block is a novel paraspinal plane block first described for thoracic analgesia [3] but is also effective in providing somatic and visceral analgesia following abdominal surgery [4]. It is relatively simple and safe to perform and thus is a feasible option where other more invasive blocks are contraindicated. We report three consecutive cases of ESP blocks performed under general anesthesia at the end of the surgery following unexpected conversion to OC via a right subcostal incision. We also describe the clinical evolution of our approach for providing optimal analgesia with the ESP block in this setting. Written Institutional Review Board consent was obtained for publication of these reports.

In the first case (a 51-year-old woman, height $165 \mathrm{~cm}$, weight $107 \mathrm{~kg}$ ), an ESP block was selected as the regional analgesia

Corresponding author: Julien Raft, M.D.

Department of Anesthesiology and Pain Medicine, University of Montréal, Maisonneuve-Rosemont Hospital, 5415 Boulevard de l'Assomption, Montréal, H1T2M4, QC, Canada

Tel: +1-514-252-3426, Fax: +1-514-252-3542

Email: julien.raft@free.fr

ORCID: https://orcid.org/0000-0001-5019-3275

Received: December 10, 2018.

Accepted: December 28, 2018.

Korean J Anesthesiol 2019 October 72(5): 504-505

https://doi.org/10.4097/kja.d.18.00350 technique because of concerns related to subcutaneous injection of $5000 \mathrm{IU}$ heparin at the beginning of surgery and the risk of bleeding complications with other options such as a thoracic paravertebral block. The patient was turned to a left lateral decubitus position. A high-frequency linear ultrasound probe 15-6 $\mathrm{MHz}$ (XPORTE, Sonosite, Canada) was placed in a parasagittal orientation over the right T5 transverse process. A $22 \mathrm{G} \times 80$ $\mathrm{mm}$ block needle (Pajunk, Germany) was inserted using an inplane, caudal-to-cranial approach. Thirty $\mathrm{ml}$ of ropivacaine 0.5\% (AstraZeneca, Canada) was injected under the erector spinae muscle. Upon arrival to the post anesthesia care unit (PACU), the patient reported diffuse abdominal pain without any particular localization to the incision site. The severity was rated as $8 / 10$ on the numeric rating scale (NRS) and a total dose of IV hydromorphone $2.5 \mathrm{mg}$ was administered to relieve this pain. Assessment of sensory block was not performed. Over the first two postoperative days (POD) the patient received oral acetaminophen 975 mg 6-hourly, celecoxib 200 mg 12-hourly, and IV patient-controlled analgesia with hydromorphone 0.2 mg every $5 \mathrm{~min}$ as needed. Total hydromorphone doses were as follows: $1.8 \mathrm{mg}$ on POD 0 (following PACU discharge), $3.6 \mathrm{mg}$ on POD 1, and $4 \mathrm{mg}$ on POD 2. Resting and dynamic NRS pain scores remained below $4 / 10$ and 5/10 respectively during this time. The patient was discharged home on POD 3.

In the second case (a 44-year-old woman, height $155 \mathrm{~cm}$, weight $90 \mathrm{~kg}$ ), the ESP block was performed in the same manner as described at the T6 transverse process, but this time bilateral blocks were performed in an attempt to improve the analgesic effect. Twenty $\mathrm{ml}$ of ropivacaine $0.5 \%$ was injected on both sides. On arrival in the PACU, the patient reported mild abdominal pain (NRS 3/10) that was again diffuse rather than localized.

(c) This is an open-access article distributed under the terms of the Creative Commons Attribution Non-Commercial License (http://creativecommons.org/ licenses/by-nc/4.0/), which permits unrestricted non-commercial use, distribution, and reproduction in any medium, provided the original work is properly cited. 
She had bilateral sensory loss to cold over T4-T9 dermatomes. Three doses of IV hydromorphone $0.2 \mathrm{mg}$ were administered in PACU to maintain pain scores $<3 / 10$. Postoperative analgesia included oral acetaminophen $975 \mathrm{mg}$ 6-hourly, oral celecoxib $200 \mathrm{mg}$ 12-hourly, and subcutaneous hydromorphone as needed. Total hydromorphone doses were as follows: $2 \mathrm{mg}$ on POD 0 (following PACU discharge) and $8 \mathrm{mg}$ on POD 1. There was no discernible sensory block to cold on POD 1 . The resting and dynamic NRS pain scores remained below 4/10 until the patient was discharged home on POD 2.

In the third case (an 86-year-old woman, height $162 \mathrm{~cm}$, weight $73 \mathrm{~kg}$ ), bilateral ESP blocks were again performed at the T6 transverse process, with injection of $15 \mathrm{ml}$ of ropivacaine $0.5 \%$ per side. A further modification to the analgesic strategy in this case was insertion of a 20-gauge catheter (Stimucath ${ }^{\circledR}$, Arrow, USA) on the right side, threaded $3 \mathrm{~cm}$ beyond the needle tip, in an effort to prolong the analgesia provided by the ESP block. In the PACU, the patient had bilateral sensory loss to cold over T4-T10 dermatomes. Postoperative analgesia comprised a continuous infusion of $0.2 \%$ ropivacaine at $10 \mathrm{ml} / \mathrm{h}$ through the right-sided ESP catheter and oral acetaminophen $975 \mathrm{mg}$ 6-hourly. On arrival in the PACU, the patient reported mild generalized abdominal discomfort of NRS 4/10 severity. A total of $0.6 \mathrm{mg}$ of IV hydromorphone was administered in PACU; however no further opioids were required during the remainder of the postoperative period. Resting and dynamic NRS pain scores were $<3 / 10$ during this time. The patient had right-sided sensory loss to cold over the T5-T9 dermatomes during the period of continuous ESP blockade. The ESP catheter was removed on POD 3 and the patient was discharged home on POD 5.

These three cases reflect the clinical evolution of the use of the ESP block in OC. In the first case, possible reasons for the suboptimal analgesia provided by the unilateral ESP block include 1) failure to cover laparoscopic incisions on the left hemi-abdomen and 2) crossover innervation of the right-sided subcostal incision [5]. To address these limitations, we performed bilateral ESP blocks in the second patient, and also tar- geted the T6 instead of the T5 transverse process as it was more congruent with the subcostal incision. This resulted in better analgesia; however there was a rebound in opioid requirements on POD 1 that coincided with the resolution of the cutaneous sensory block. We therefore inserted a right-sided ESP catheter in the third case to prolong regional analgesia that provided excellent pain relief with minimal opioid requirements. In summary, our experience indicates that probably bilateral ESP blocks are required to cover unilateral incisions extending medial to the mid-clavicular line, and that a continuous catheter technique may provide optimal analgesia where moderate-to-severe postoperative pain is expected beyond 24 hours.

\section{Conflicts of Interest}

No potential conflict of interest relevant to this article was reported.

\section{Author Contributions}

Julien Raft (Conceptualization; Supervision; Validation; Visualization; Writing-original draft)

Ki Jinn Chin (Conceptualization; Supervision; Validation; Visualization; Writing-original draft)

Quentin Gobert (Conceptualization; Supervision; Validation; Visualization; Writing-original draft)

Philippe Richebé (Conceptualization; Supervision; Validation; Visualization; Writing-original draft)

Véronique Brulotte (Conceptualization; Supervision; Validation; Visualization; Writing-original draft)

\section{ORCID}

Julien Raft, https://orcid.org/0000-0001-5019-3275

Ki Jinn Chin, https://orcid.org/0000-0002-8339-3764

Philippe Richebé, https://orcid.org/0000-0003-2808-9660

Véronique Brulotte, https://orcid.org/0000-0001-8901-8554

\section{References}

1. Kama NA, Kologlu M, Doganay M, Reis E, Atli M, Dolapci M. A risk score for conversion from laparoscopic to open cholecystectomy. Am J Surg 2001; 181: 520-5.

2. Hendolin HI, Pääkönen ME, Alhava EM, Tarvainen R, Kemppinen T, Lahtinen P. Laparoscopic or open cholecystectomy: a prospective randomised trial to compare postoperative pain, pulmonary function, and stress response. Eur J Surg 2000; 166: 394-9.

3. Forero M, Adhikary SD, Lopez H, Tsui C, Chin KJ. The erector spinae plane block: a novel analgesic technique in thoracic neuropathic pain. Reg Anesth Pain Med 2016; 41: 621-7.

4. Restrepo-Garces CE, Chin KJ, Suarez P, Diaz A. Bilateral continuous erector spinae plane block contributes to effective postoperative analgesia after major open abdominal surgery: a case report. A A Case Rep 2017; 9: 319-21.

5. Capek S, Tubbs RS, Spinner RJ. Do cutaneous nerves cross the midline? Clin Anat 2015; 28: 96-100. 